\title{
A Quantitative Model of Glucose Signaling in Yeast Reveals an Incoherent Feed Forward Loop Leading to a Specific, Transient Pulse of Transcription
}

\author{
Sooraj KuttyKrishnan ${ }^{1}$, Jeffrey Sabina ${ }^{2}$, Laura Langton ${ }^{3}$, \\ Mark Johnston ${ }^{4}$, and Michael R. Brent ${ }^{3}$ \\ 1 University of Washington \\ 2 Ion Torrent \\ 3 Washington University \\ 4 University of Colorado Denver
}

The ability to design and engineer organisms demands the ability to predict kinetic responses of novel regulatory networks built from well-characterized biological components. Surprisingly, few validated kinetic models of complex regulatory networks have been derived by combining models of the network components. A major bottleneck in producing such models is the difficulty of measuring in vivo rate constants for components of complex networks. We demonstrate that a simple, genetic approach to measuring rate constants in vivo produces an accurate kinetic model of the complex network that Saccharomyces cerevisiae employs to regulate the expression of genes encoding glucose transporters. The model predicts a transient pulse of transcription of HXT4 (but not HXT2 or HXT3) in response to addition of a small amount of glucose to cells, an outcome we observed experimentally. Our model also provides a mechanistic explanation for this result: HXT24 are governed by a type 2, incoherent feed forward regulatory loop involving the Rgt1 and Mig2 transcriptional repressors. The efficiency with which Rgt1 and Mig2 repress expression of each HXT gene determines which of them have a pulse of transcription in response to glucose. Finally, the model correctly predicts how lesions in the feed forward loop change the kinetics of induction of HXT4 expression. 